\title{
Prostatectomía radical laparoscópica versus abierta
}

\author{
Raventós Busquets CX, Gómez Lanza E, Cecchini Rosell L, Trilla Herrera E, \\ Orsola de los Santos A, Planas Morin J, De Torre Ramírez I*, Morote Robles J.
}

Servicio de Urología. *Servicio de Anatomía Patológica. Hospital General Universitari Vall d'Hebron. Barcelona.

Actas Urol Esp. 2007; 31(2):141-145

\begin{abstract}
RESUMEN
PROSTATECTOMÍA RADICAL LAPAROSCÓPICA VERSUS ABIERTA

Objetivos. Analizar las diferencias entre la prostatectomía radical abierta (PRA) y la prostatectomía radical laparoscópica (PRL).

Material y métodos. Se evalúan 180 cirugías prostáticas radicales realizadas en el 2004 y 2005, de las cuales 105 (58\%) fueron laparoscópicas (PRL) y 75 (42\%) por vía abierta. Más de la mitad de las intervenciones se realizaron por cirujanos expertos: 51\% en las PRL y 56\% en las PRA. Se compararon las variables: tiempo quirúrgico, sangrado intraoperatorio (diferencia entre hematocrito prequirúrgico y postquirúrgico) y los días de ingreso. El control oncológico y quirúrgico se evaluaron mediante los parámetros: margen maligno (MM) (margen positivo en un pT3) e incisión quirúrgica benigna (IQB) y maligna (IQM).

Resultados. Los dos grupos son comparables respecto a la edad, estadio clínico, Gleason de la biopsia y volumen, sólo difiriendo en el valor de PSA. La media del tiempo quirúrgico fue significativamente mayor en la PRL (172 minutos) versus la PRA (145 minutos). La diferencia del hematocrito fue mayor en la PRA (10,7 puntos) respecto a la PRL (9,2 puntos) ( $\mathrm{p}=0,03$ ), así como los días de ingreso, representando un día más en la PRA ( $\mathrm{p}=0,001)$. Un 26,7\% de las PRL han presentado IQB, frente a un 48,7 \% en las PRA ( $\mathrm{p}=0,001$ ). Desde el punto de vista oncológico se han observado un $5,4 \%$ de MM en las PRL versus un 16,9\% en las PRA. (p=0,023). No obstante, no se han observado diferencias respecto a las IQM.

Conclusión. En la PRL, a igualdad de estadio clínico y patológico, se observa un mejor control de los márgenes junto con una menor afectación quirúrgica de la pieza. También existe una menor pérdida sanguínea así como una reducción de los días de ingreso. Por tanto, se puede concluir que la PRL es, en nuestro entorno, una técnica válida para el tratamiento del cáncer de próstata organoconfinado, a pesar de un mayor tiempo quirúrgico (27 minutos) y de la dificultad de su aprendizaje.
\end{abstract}

Palabras clave: Cáncer de próstata. Laparoscopia. Prostatectomía radical.

\section{ABSTRACT}

COMPARISON BETWEEN OPEN AND LAPAROSCOPIC APPROACH IN RADICAL PROSTATECTOMY

Introduction. To evaluate the differences between laparoscopic (LRP) and open radical prostatectomy (ORP).

Material and Methods. From 2004 to 2005180 Radical prostatectomies (RP) were performed, 105 laparoscopical and 75 by an open approach. Different urologists have acted as first surgeon; 51\% of them, fully experienced ones in OPR, and 56\% in LRP. Differences in operative time, estimated blood loss (difference of pre and post operative hematocrite), and duration of hospitalization were compared. Additionally, we have also analysed surgical and oncologic control of the specimen defined by the following variables: Malignant margins (MM) (positive margin in a pT3 specimen), and benign/malign surgical incision (BSI/MSI).

Results. Groups were similar concerning age, clinical stage and Gleason score, and there are only differences in PSA. Mean operative time was significantly higher in LRP (172 minutes) versus ORP (145 minutes) ( $<<0.001)$. Difference of pre and post operative hematocrite was also higher in the open group (10.7 vs 9,2) ( $\mathrm{p}=0.03)$, together with hospital stay, which was one day longer in the ORP group ( $\mathrm{p}=0.001)$. ORP group had a higher rate of benign surgical incisions $(48.7 \%$ vs $26.7 \%)$ ( $\mathrm{p}=0.001)$. Regarding oncologic results, LRP presented a $5.4 \%$ of positive margins, which compared significantly with a $16.9 \%$ rate in the open group $(\mathrm{p}=0.023)$. However, no differences concerning malignant surgical incisions were observed.

Conclusion. With no differences in clinical and pathological stage, LRP offers a significant reduction of surgical aggressiveness on the specimen, together with a better MM control. We also observe a clear decrease in blood loss and hospital stay. Therefore, we conclude that LRP in our environment is a valid approach of surgical prostate cancer treatment in spite of a longer operative time (27 minutes) and a steep learning curve.

Keywords: Prostate cancer. Laparoscopy. Radical prostatectomy. 
$\mathrm{L}^{2}$ a prostatectomía radical abierta (PRA) se considera el tratamiento de elección del cáncer de próstata $^{1}$, cuyos resultados han mejorado gracias a los progresos en la técnica quirúrgica, el manejo postoperatorio así como a una correcta selección de los pacientes. La introducción de la cirugía laparoscópica en la Urología y en concreto la estandarización de la prostatectomía radical laparoscópica (PRL) invita a la comparación entre las dos técnicas quirúrgicas. La alta incidencia del cáncer de próstata así como del gran interés de la comunidad urológica en la cirugía laparoscópica ha conseguido igualar o incluso mejorar los resultados como se refleja en algunos trabajos publica$\operatorname{dos}^{2}$. En este estudio comparamos de manera prospectiva los resultados intra y preoperatorios entre la PRA y la PRL con objetivo de evaluar posibles ventajas de la técnica laparoscópica.

\section{MATERIAL Y MÉTODOS}

De enero de 2004 a enero de 2006 se practicaron 180 cirugías prostáticas radicales consecutivas, de las cuales 105 se realizaron por vía laparoscópica y 75 por vía abierta. Un 51\% de los casos se realizaron por cirujanos experimentados en las PRA y en un 56\% de las PRL.

La técnica realizada en la PRA fue la descrita por Walsh en el $2000^{1}$, sin añadir linfadenectomía en el 41\%. En cuanto a la técnica quirúrgica laparoscópica se realizó inicialmente la descrita por el grupo de Montsouris ${ }^{3}$, con alguna modificación y obviando la disección de la vía seminal en el fondo de saco de Douglas. De las PRL, 20 se realizaron vía extraperitoneal, que reproduce la misma técnica y que tiene clara utilidad en los casos con cirugía abdominal previa. Es la técnica utilizada habitualmente en nuestro centro por permitir un más rápido acceso y reducir el tiempo quirúrgico.

Las variables que se analizan para comparar ambos grupos son: edad (años), PSA, volumen prostático por ecografía, estadio clínico y Gleason de la biopsia. El control quirúrgico se evalúa mediante los parámetros de incisión quirúrgica benigna (IQB) y maligna (IQM) y el control oncológico mediante el margen maligno (MM). Se definen como IQB aquellas incisiones que irrumpen la cápsula sin afectar al tumor, las IQM son aquellas que inciden al tumor que se encuentra intra- prostático y MM son aquellos márgenes con un tumor ya extraprostático. Tradicionalmente, la suma de la incisión quirúrgica maligna y el margen maligno se considera como margen positivo (Fig. 1). Las variables clinicas que se utilizan son el tiempo quirúrgico (minutos), sangrado intraoperatorio (calculado como la diferencia entre el hematocrito prequirúrgico y el postquirúrgico) y la estancia hospitalaria en días.

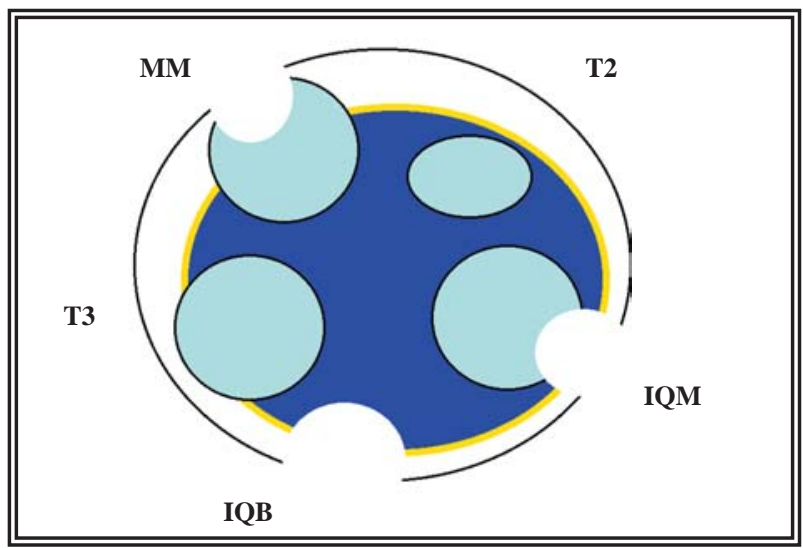

FIGURA 1. Dibujo de la glándula prostática donde se representa la Incisión Quirúrgica Benigna (IQB), la Incisión Quirúrgica Maligna (IQM) y los Márgenes Malignos (MM).

En el análisis estadístico, se utiliza el test de la chi-cuadrado para las variables categóricas y la t-student para la comparación de medias, acompañadas de su desviación estándar (SD). El valor de $\mathrm{p}<0,05$ se ha considerado significativo.

\section{RESULTADOS}

Los dos grupos fueron comparables en términos de edad, volumen prostático, estadio Gleason de la biopsia y estadio clínico. No obstante encontramos diferencias significativas en cuanto al PSA [en la PRA, PSA=9,28 ng/ml y en la PRL, $\mathrm{PSA}=7,1 \mathrm{ng} / \mathrm{ml}(\mathrm{p}=0,001)$ ] (Tabla 1$)$.

Tabla 1

\begin{tabular}{lccc}
\hline & Abierta & Laparoscópica & p \\
\hline Edad (SD) & $65,6(6,7)$ & $65(5,9)$ & $\mathrm{ns}$ \\
PSA (SD) & 9,28 & $7,1(2,2)$ & 0,001 \\
Volumen (SD) & $47,3(28,8)$ & $40,2(20,4)$ & $\mathrm{ns}$ \\
Gleason (\%) & & & \\
$\quad$ < 6 & 53 & 52,6 & $\mathrm{~ns}$ \\
$\quad>6$ & 47 & 47,4 & $\mathrm{~ns}$ \\
Estadio clínico (\%) & & & \\
$\quad$ T1 & 76,9 & 74 & $\mathrm{~ns}$ \\
$\quad$ T2 & 23,1 & 26 & $\mathrm{~ns}$ \\
\hline
\end{tabular}


La media del tiempo quirúrgico (+/- SD) en la cirugía abierta fue de 145,1+/-32,9 minutos. Con la técnica laparoscópica se tardaron 27,2 minutos más (172,3+/-43,7), parámetro estadísticamente significativo $(\mathrm{p}<0,0001)$ (Tabla 2). En cuanto al sangrado intraoperatorio, se observó que en la PRL la diferencia del hematocrito pre y postquirúrgico fue de 9,2\% mientras que en la cirugía abierta se produjo un mayor descenso del 10,7 \% ( $\mathrm{p}=0,03)$. La estancia media hospitalaria fue un día más larga en la PRA, siendo de 4,8 días en la PRL y de $5,79$ en la PRA ( $p=0,001)$ (Tabla 2).

$\mathrm{El}$ porcentaje de incisiones quirúrgicas benignas en la PRA fue del 48,7\%, superior al 26,7 \% de la PRL ( $\mathrm{p}=0,001)$; asimismo se observaron valores algo mayores de incisiones quirúrgicas malignas en la PRA, 21,5\% frente al $15 \%$ de la PRL $(p=0,25)$. En cuanto a las PRA, un 14\% presentaban los dos tipos de incisiones quirúrgicas a la vez, frente a un 5,7\% de las PRL ( $p=0,08)$. Respecto al estadio patológico, se observaron 70,9 \% de tumores organoconfinados (pT2) en la PRA y un $80 \%$ en la PRL; y con afectación extracapsular (pT3) un 29,1\% en la PRA respecto a un $20 \%$ en la cirugía laparoscópica, no siendo este último dato significativo $(\mathrm{p}=0,053)$ (Tabla 3). En

Tabla 2

\begin{tabular}{lccc}
\hline & Abierta & Laparoscópica & p \\
\hline Tiempo quirúrgico (min) (SD) & $145,1(32,9)$ & $172,3(43,7)$ & $<0,0001$ \\
$\begin{array}{l}\text { Sangrado intraoperatorio (SD) } \\
\text { (Ht pre-Ht post) }\end{array}$ & $10,7(3,9)$ & $9,2(4,4)$ & 0,03 \\
Estancia hospitalaria (días) (SD) & $5,79(1,67)$ & $4,8(1,3)$ & 0,001 \\
\hline
\end{tabular}

Tabla 3

\begin{tabular}{llccc}
\hline & & Abierta & Laparoscópica & p \\
\hline Estadio patológico (\%) & pT2 & 70,9 & 80 & 0,053 \\
& pT3 & 29,1 & 20 & \\
Incisión quirúrgica benigna (\%) & NO & 51,3 & 73,3 & 0,001 \\
& SI & 48,7 & 26,7 & \\
Incisión quirúrgica maligna (\%) & NO & 78,5 & 85 & 0,25 \\
& SI & 21,5 & 15 & \\
Márgenes malignos (\%) & - & 83,5 & 94,3 & 0,026 \\
Margen global (\%) & + & 16,5 & 5,7 & \\
\hline
\end{tabular}

los pT3, se detectó una mayor afectación de márgenes malignos en la PRA (16,5\%) que en la PRL $(5,7 \%) \quad(p=0,026)$. El porcentaje de márgenes malignos tal y como se considera en la literatura era del 38\% en la PRA (21,5\% pT2 + 16,5\% pT3) y del $20,7 \%$ en la PRL (15\% pT2 + 5,7\% pT3) $(\mathrm{p}=0,03)$.

\section{DISCUSIÓN}

En este estudio, a igualdad de estadio clínico y patológico, observamos un mejor control de los márgenes como resultado en parte de una menor agresividad sobre la pieza. El tiempo quirúrgico en la cirugía laparoscópica supone media hora más y los resultados postoperatorios desde el punto de vista de sangrado y estancia son mejores.

Cabe destacar las diferencias significativas en cuanto a los niveles medios de PSA entre los dos grupos. Esto lo atribuimos a un mayor volumen prostático en el grupo de las PRA, debido a la selección en el inicio de la cirugía laparoscópica en nuestro centro de aquellas próstatas "no grandes". Si observamos la mediana entre los dos grupos, ésta no varía (PSA: 6,4 ng/ml en la PRA y 6,6 $\mathrm{ng} / \mathrm{ml}$ en la PRL).

En nuestro centro se ha aplicado un sistema de aprendizaje mediante la figura de un tutor que ha facilitado la reproducibilidad de la técnica en un corto periodo de tiempo. Este modelo ha permitido una buena curva de aprendizaje que se ha traducido en un tiempo quirúrgico realmente competitivo. Por otro lado parece más importante la experiencia del cirujano que el tipo de técnica laparoscópica a elegir.

El sangrado intraoperatorio es menor en la PRL respecto a la PRA, requiriendo menor porcentaje de transfusiones. Estos resultados son comparables a series ya publicadas ${ }^{4,5}$. En cuanto a los días de ingreso, los resultados en la literatura pueden ser paradójicos según el sistema sanitario del país, siendo muy 
bajos en EEUU (PRA) y altos en Francia (PRL) ${ }^{6,7}$. En nuestro caso la cirugía laparoscópica ahorra un día de ingreso.

En cuanto a la agresividad sobre la pieza quirúrgica, ésta es claramente superior en la PRA observando más disrupciones capsulares benignas. Hemos intentado diferenciar la presencia de incisiones quirúrgicas tumorales de los márgenes tumorales en estadios pT3. Podríamos considerar asimismo las incisiones quirúrgicas malignas como traducción de la calidad de la cirugía. El aumento de la visión, junto con la mejor manipulación de la pieza creemos que puede evitar la presencia de incisiones quirúrgicas así como, porqué no, de los márgenes positivos en los pT3, mejorando los resultados oncológicos. En nuestra serie, si bien es cierto que el porcentaje de pT3 es mayor en la PRA $(29,5 \%$ vs $20 \%$, p $=0,053)$, los márgenes positivos en este grupo son casi el doble a lo que deberíamos esperar $(16,5 \%$ vs $5,7 \%$ ), este hecho junto con la mayor presencia de incisiones quirúrgicas malignas $(21,5 \%$ PRA, $14 \%$ PRL) aumenta la presencia de márgenes positivos en la PRA, tal y como se consideran en la literatura (margen global).

En muchos artículos donde se comparan ambas cirugías, éstas son realizadas en diferentes centros o con cirujanos distintos o en diferentes periodos de experiencia quirúrgica en cáncer de próstata ${ }^{8-12}$. Es en las referencias donde el mismo equipo de urólogos ya expertos en ambos abordajes y que los realizan de manera rutinaria, cuando se observan unos resultados favorables para la PRL ${ }^{13}$.

Las dos técnicas quirúrgicas comparten tanto la indicación como las contraindicaciones. El volumen prostático que inicialmente limitaba la actuación laparoscópica ha dejado de influir. La PRA, es considerada como el gold standard del tratamiento del cáncer de próstata localizado, con resultados reproducibles desde el punto de vista funcional y oncológico. La PRL es una técnica joven ( 7 años) con un futuro prometedor al haber conseguido ya los resultado de la PRA pero siendo todavía una técnica en desarrollo. La miniinvasividad de la PRL ligada a la exquisitez de la disección anatómica son sus principales atributos que junto al desarrollo de su instrumental permitirá nuevos horizontes.
En nuestro centro ante estos resultados se ha apostado por la cirugía laparoscópica del cáncer de próstata, tal como se refleja en la actividad de estos dos años (Fig. 2). En nuestra opinión la PRL posee ventajas que hacen prever mejores resultados en continencia y recuperación de la erección a largo plazo.

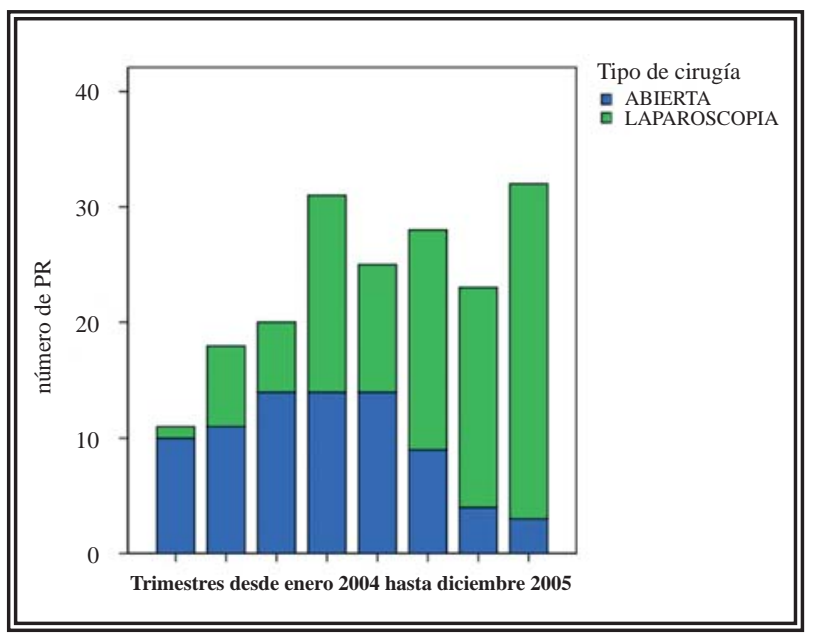

FIGURA 2. Evolución a lo largo de los años 2004-2005 del número de prostatectomias radicales abiertas y laparoscópicas.

\section{CONCLUSIÓN}

En la PRL se observa un mejor control de los márgenes junto con una menor afectación quirúrgica de la pieza. También existe una menor pérdida sanguínea así como una reducción de los días de ingreso. Por tanto, se puede concluir que la PRL es, en nuestro entorno, una técnica válida y una atractiva alternativa para el tratamiento del cáncer de próstata órgano-confinado, a pesar de un mayor tiempo quirúrgico (27 minutos) y de la dificultad de su aprendizaje.

\section{REFERENCIAS}

1. Walsh PC. Radical prostatectomy for localized prostate cancer provides durable cancer control with excellent quality of life: a structured debate. J Urol. 2000;163(6):1802-1807.

2. Hoznek A, Menard Y, Salomon L, Abbou CC. Update on laparoscopic and robotic radical prostatectomy. Curr Opin Urol. 2005; 15(3):173-180.

3. Guillonneau B, Vallancien G: Laparoscopic radical prostatectomy: the Montsouris technique. J Urol. 2000;163(6): 1643-1649.

4. Davis JW, Kuban DA, Lynch DF, Schellhammer PF. Quality of life after treatment for localized prostate cancer: differences based on treatment modality. J Urol. 2001;166(3): 947952. 
5. Remzi M, Klingler HC, Tinzl MV, Fong YK, Lodde M, Kiss B et al. Morbidity of laparoscopic extraperitoneal versus transperitoneal radical prostatectomy verus open retropubic radical prostatectomy. Eur Urol. 2005,48(1):83-89;discussion 89

6. Salomon L, Levrel O, De la Taille A, Anastasiadis AG, Saint $\mathrm{F}$, Zaki $\mathrm{S}$ et al. Radical prostatectomy by the retropubic, perineal and laparoscopic approach: 12 years of experience in one center. Eur Urol. 2002;42(2):104-110;discussion 110-101.

7. Salomon L, Sebe P, De La Taille A, Vordos D, Hoznek A, Yiou $\mathrm{R}$ et al. Open versus laparoscopic radical prostatectomy: Part II. BJU Int. 2004;94(2):244-250.

8. Artibani W, Grosso G, Novara G, Pecoraro G, Sidoti O, Sarti A et al. Is laparoscopic radical prostatectomy better than traditional retropubic radical prostatectomy? An analysis of peri-operative morbidity in two contemporary series in Italy. Eur Urol. 2003;44(4):401-406.

9. Rassweiler J, Seemann O, Schulze M, Teber D, Hatzinger M, Frede T. Laparoscopic versus open radical prostatectomy: a comparative study at a single institution. J Urol. 2003;169 (5): 1689-1693
10. El-Feel A, Davis JW, Deger S, Roigas J, Wille AH, Schnorr D et al. Laparoscopic radical prostatectomy-an analysis of factors affecting operating time. Urology 2003;62(2):314-318.

11. Anastasiadis AG, Salomon L, Katz R, Hoznek A, Chopin D, Abbou CC. Radical retropubic versus laparoscopic prostatectomy: a prospective comparison of functional outcome. Urology 2003;62(2):292-297.

12. Guillonneau B, Vallancien G. Laparoscopic radical prostatectomy: the Montsouris experience. J Urol. 2000;163(2): 418-422.

13. Guazzoni G, Cestari A, Naspro R, Riva M, Centemero A, Zanoni $\mathrm{M}$ et al. Intra- and peri-operative outcomes comparing radical retropubic and laparoscopic radical prostatectomy: results from a prospective, randomised, single-surgeon study. Eur Urol. 2006;50(1):98-104.

Correspondencia autor: Dr. C.X. Raventós Busquets

Servicio de Urología. Hospital Vall d'Hebrón. Passeig de la Vall d'Hebron 119-129. 08054 Barcelona. Tel.: 932746000 Ext 6370 E-mail autor: cravento@vhebron.net

Información articulo: Original - Laparoscopia

Trabajo recibido: septiembre 2006

Trabajo aceptado: octubre 2007 